\title{
DARK AND SINGULAR DISPERSIVE OPTICAL SOLITONS OF SCHRÖDINGER-HIROTA EQUATION BY MODIFIED SIMPLE EQUATION METHOD
}

\author{
Ahmed H. Arnous ${ }^{1}$, Malik Zaka Ullah ${ }^{2}$, Mir Asma ${ }^{3}$, Seithuti P. Moshokoa ${ }^{4}$, \\ Qin Zhou ${ }^{5}$, Mohammad Mirzazadeh ${ }^{6}$, Anjan Biswas ${ }^{2,4} \&$ Milivoj Belic ${ }^{7}$ \\ 1 Department of Engineering Mathematics and Physics, \\ Higher Institute of Engineering, El-Shorouk, Cairo, Egypt. \\ ${ }^{2}$ Department of Mathematics, Faculty of Science, \\ King Khalid University, PO Box 9004, Abha-61413, Saudi Arabia \\ 3 Institute of Mathematical Sciences, Faculty of Science, \\ University of Malaya, 50601 Kuala Lumpur, Malaysia \\ 4 Department of Mathematics and Statistics, \\ Tshwane University of Technology, Pretoria-0008, South Africa \\ 5 School of Electronics and Information Engineering, \\ Wuhan Donghu University, Wuhan, 430212, PR China \\ ${ }^{6}$ Department of Engineering Sciences, \\ Faculty of Technology and Engineering, East of Guilan, \\ University of Guilan, P.C. 44891-63157, Rudsar-Vajargah, Iran \\ 7 Science Program, Texas A \& M University at Qatar, \\ PO Box 23874, Doha, Qatar
}

\begin{abstract}
This paper obtains dispersive dark and singular optical solitons, governed by Schrödinger-Hirota equation, in optical fibers. The integration algorithm is the modified simple equation method. Both Kerr law and power laws of nonlinearity are considered.
\end{abstract}

Keywords: solitons, Schrödinger-Hirota equation; modified simple equation method. 


\section{INTRODUCTION}

Dispersive optical solitons are studied when group velocity dispersion (GVD) is small and needs to be supplemented with third order dispersion (3OD) [1-20]. The model that accuretely describes this phenomena is the Schrödinger-Hirota equation (SHE) which stems from the familiar nonlinear Schrödinger's equation (NLSE) through Lie transform. This derivation is described in lot of earlier works [4-10]. The integration tool that will be applied to SHE is the modified simple equation method. This scheme will lead to the extraction of dark and singular optical solitons only. Both Kerr and power laws of nonlinearity are studied in this paper. It will also be proved that power law of nonlinearity will not support these soliton solutions. The constraint conditions for the existence of such solitons in Kerr law medium will be presented.

\section{THE MODIFIED SIMPLE EQUATION METHOD (SUCCNCT REVIEW)}

Suppose we have a nonlinear evolution equation in the form

$$
P\left(u, u_{t}, u_{x}, u_{x x}, u_{t t}, u_{t x}, \ldots\right)=0,
$$

where $P$ is a polynomial in $u(x, t)$ and its partial derivatives in which the highest order derivatives and nonlinear terms are involved. In the following, we give the main steps of this method $[1,19,20]$.

Step-1: We use the transformation

$$
u(x, t)=u(\xi), \quad \xi=x-c t,
$$

where $c$ is a constant to be determined, to reduce Eq. (1) to the following ODE :

$$
Q\left(u, u^{\prime}, u^{\prime \prime}, \ldots\right)=0,
$$

where $Q$ is a polynomial in $u(\xi)$ and its total derivatives, while ${ }^{\prime}=\frac{d}{d \xi}$.

Step-2: We suppose that Eq. (3) has the formal solution.

$$
u(\xi)=\sum_{l=0}^{N} a_{l}\left(\frac{\psi^{\prime}(\xi)}{\psi(\xi)}\right)^{l},
$$

where $a_{l}$ are constants to be determined, such that $a_{N} \neq 0$, and $\psi(\xi)$ is an unknown function to be determined later.

Step-3: We determine the positive integer $N$ in Eq. (4) by considering the homogeneous balance between the highest order derivatives and the nonlinear terms in Eq. (3).

Step-4: We substitute (4) into (3), then we calculate all the necessary derivatives $u^{\prime}, u^{\prime \prime}, \ldots$ of the unknown function $u(\xi)$ and we account the function $\psi(\xi)$. As a result of this substitution, we get a polynomial of $\psi^{\prime}(\xi) / \psi(\xi)$ and its derivatives. In this polynomial, we gather all the terms of the same power of $\psi^{-j}(\xi), j=0,1,2, \ldots$ and its derivatives, and we equate with zero all the coefficients of this polynomial. This operation yields a system of equations which can be solved to find $a_{k}$ and $\psi(\xi)$. Consequently, we can get the exact solutions of Eq. (1) . 


\section{APPLICATION TO SHE}

This section will implement modified simple equation method to SHE for two types of nonlinear media. The details are described in subsequent subsections.

\subsection{KERR LAW NONLINEARITY}

The dimensionless form of the perturbed SHE with STD and Kerr law nonlinearity is given by [4-6, 8-10, 12]

$$
i q_{t}+a q_{x x}+b q_{x t}+c|q|^{2} q+i\left(\gamma q_{x x x}+\sigma|q|^{2} q_{x}\right)=i \alpha q_{x}+i \lambda\left(|q|^{2} q\right)_{x}+i \nu\left(|q|^{2}\right)_{x} q
$$

where $a$ represents the GVD and $b$ is the spatio-temporal dispersion (STD) term. The inclusion of STD makes the model well-posed as suggested during $2012[14,15]$. From perturbation terms, $\gamma$ represents the 3OD coefficient, $\nu$ and $\sigma$ represent the nonlinear dispersion, $\alpha$ the inter-modal dispersion and $\lambda$ the self steepening term.

In order to solve this equation by using the modified simple equation method, the following solution structure is selected

$$
q(x, t)=U(\xi) e^{i \Phi(x, t)},
$$

where the wave variable $\xi$ is given by

$$
\xi=k(x-v t)
$$

Here, $U(\xi)$ represents the amplitude component of the soliton solution and $v$ is the speed of the soliton, while the phase component $\Phi(x, t)$ is defined as

$$
\Phi(x, t)=-\kappa x+\omega t+\theta,
$$

where $\kappa$ is the frequency of the soliton, $\omega$ is the wave number, while $\theta$ is the phase constant.

Substituting (6) into (5) and decomposing into real and imaginary parts lead to

$$
\begin{gathered}
k^{2} U^{\prime \prime}(a-b v+3 \gamma \kappa)-\left(a \kappa^{2}+\alpha \kappa+\omega(1-b \kappa)+\gamma \kappa^{3}\right) U+(c-\kappa \lambda+\kappa \sigma) U^{3}=0, \\
\gamma k^{3} U^{\prime \prime}-k\left(2 a \kappa+\alpha+v(1-b \kappa)-b \omega-3 \gamma \kappa^{2}\right) U+\frac{1}{3} k(\sigma-3 \lambda-2 \nu) U^{3}=0 .
\end{gathered}
$$

Balancing $U^{\prime \prime}$ with $U^{3}$ in Eqs. (9) and (10), then we get $N=1$. Consequently we reach

$$
U(\xi)=a_{0}+a_{1}\left(\frac{\psi^{\prime}(\xi)}{\psi(\xi)}\right), \quad a_{1} \neq 0 .
$$

Substituting Eq. (11) in Eqs. (9) and (10) and then setting the coefficients of $\psi^{-j}(\xi), j=0,1,2,3$, to zero, then we obtain a set of algebraic equations involving $a_{0}, a_{1}, k, \kappa, \gamma, \alpha, \nu, v$ and $\omega$ as follows:

$\psi^{-3}$ coeff.:

$$
\begin{gathered}
a_{1}\left(\psi^{\prime}\right)^{3}\left(2 k^{2}(a-b v+3 \gamma \kappa)+a_{1}^{2}(c+\kappa(\sigma-\lambda))\right)=0, \\
\frac{1}{3} a_{1} k\left(\psi^{\prime}\right)^{3}\left(a_{1}^{2}(-3 \lambda-2 \nu+\sigma)+6 \gamma k^{2}\right)=0,
\end{gathered}
$$

$\psi^{-2}$ coeff.:

$$
\begin{gathered}
3 a_{1} \psi^{\prime}\left(a_{0} a_{1} \psi^{\prime}(c+\kappa(\sigma-\lambda))-k^{2} \psi^{\prime \prime}(a-b v+3 \gamma \kappa)\right)=0, \\
a_{1} k \psi^{\prime}\left(a_{0} a_{1} \psi^{\prime}(-3 \lambda-2 \nu+\sigma)-3 \gamma k^{2} \psi^{\prime \prime}\right)=0,
\end{gathered}
$$


$\psi^{-1}$ coeff.:

$$
\begin{gathered}
-a_{1}\left(\psi^{\prime}\left(-3 a_{0}^{2}(c-\kappa \lambda+\kappa \sigma)+a \kappa^{2}+\alpha \kappa-b \kappa \omega+\gamma \kappa^{3}+\omega\right)-k^{2} a-b v+3 \gamma \kappa \psi^{\prime \prime \prime}\right)=0, \\
k a_{1}\left(\psi^{\prime}\left(-2 a \kappa+a_{0}^{2}(-3 \lambda-2 \nu+\sigma)-\alpha+b \kappa v+b \omega+3 \gamma \kappa^{2}-v\right)+k^{2} \gamma \psi^{\prime \prime \prime}\right)=0,
\end{gathered}
$$

$\psi^{0}$ coeff.:

$$
\begin{gathered}
-a_{0}\left(-a_{0}^{2}(c+\kappa(\sigma-\lambda))+a \kappa^{2}+\alpha \kappa-b \kappa \omega+\gamma \kappa^{3}+\omega\right)=0, \\
-\frac{1}{3} a_{0} k\left(3\left(2 a \kappa+\alpha-b \kappa v-b \omega-3 \gamma \kappa^{2}+v\right)+a_{0}^{2}(3 \lambda+2 \nu-\sigma)\right)=0 .
\end{gathered}
$$

Solving this system, we obtain

$$
\begin{gathered}
\sqrt{\frac{(\sigma-3 \lambda-2 \nu)\left(b^{2} \kappa^{2}\left(\frac{\alpha}{\kappa}+a+\gamma \kappa\right)+a(1-b \kappa)^{2}\right)-(1-b \kappa)^{2}\left(3 \gamma(c+2 \kappa(\lambda+\nu))-\frac{b}{(1-b \kappa)}(\sigma-3 \lambda-2 \nu)\left(2 a \kappa+\alpha-3 \gamma \kappa^{2}\right)\right)}{\frac{1}{3} b(\sigma-3 \lambda-2 \nu)(3 b(c-\kappa \lambda+\kappa \sigma)+(1-b \kappa)(\sigma-3 \lambda-2 \nu))}}, \\
a_{1}= \pm \sqrt{\frac{6 \gamma k^{2}}{3 \lambda+2 \nu-\sigma}}, \\
v=\frac{a(\sigma-3 \lambda-2 \nu)-3 \gamma(c+2 \kappa \lambda+2 \kappa \nu)}{b(\sigma-3 \lambda-2 \nu)} \\
\omega=\frac{1}{b(-3 \lambda-2 \nu+\sigma)((b \kappa-1)(-3 \lambda-2 \nu+\sigma)-3 b(c-\kappa \lambda+\kappa \sigma))}(b(-3 \lambda-2 \nu+\sigma) \\
\left((-3 \lambda-2 \nu+\sigma)\left(a \kappa^{2}+\alpha \kappa+\gamma \kappa^{3}\right)-3\left(2 a \kappa+\alpha-3 \gamma \kappa^{2}\right)(c-\kappa \lambda+\kappa \sigma)\right)+ \\
(b \kappa-1)(3 a(-3 \lambda-2 \nu+\sigma)(c-\kappa \lambda+\kappa \sigma)-9 \gamma(c+2 \kappa \lambda+2 \kappa \nu)(c-\kappa \lambda+\kappa \sigma)+2 \kappa \lambda+2 \kappa \nu)),
\end{gathered}
$$

and

$$
\begin{gathered}
\psi^{\prime \prime}= \pm \sqrt{-\frac{2 a_{0}^{2}(\sigma-3 \lambda-2 \nu)}{3 \gamma k^{2}}} \psi^{\prime} . \\
\psi^{\prime \prime \prime}=-\frac{2 a_{0}^{2}(\sigma-3 \lambda-2 \nu)}{3 \gamma k^{2}} \psi^{\prime} .
\end{gathered}
$$

From Eqs. (24) and (25), we can deduce that

$$
\begin{aligned}
& \psi^{\prime}= \pm \sqrt{-\frac{3 \gamma k^{2}}{2 a_{0}^{2}(\sigma-3 \lambda-2 \nu)}} k_{1} e^{ \pm \sqrt{-\frac{2 a_{0}^{2}(\sigma-3 \lambda-2 \nu)}{3 \gamma k^{2}}} \xi}, \\
& \psi=-\frac{3 \gamma k^{2}}{2 a_{0}^{2}(\sigma-3 \lambda-2 \nu)} k_{1} e^{ \pm \sqrt{-\frac{2 a_{0}^{2}(\sigma-3 \lambda-2 \nu)}{3 \gamma k^{2}}} \xi}+k_{2} .
\end{aligned}
$$

where $k_{1}$ and $k_{2}$ are constants of integration. Substituting Eq. (26) and Eq. (27) into Eq. (11), we obtain following the following exact solution to Eq. (5).

$$
q(x, t)=\left\{a_{0}+\frac{\frac{3 \gamma k^{2}}{a_{0}(\sigma-3 \lambda-2 \nu)} k_{1} e^{ \pm \sqrt{-\frac{2 a_{0}^{2}(\sigma-3 \lambda-2 \nu)}{3 \gamma k^{2}}}(k(x-v t))}}{-\frac{3 \gamma k^{2}}{2 a_{0}^{2}(\sigma-3 \lambda-2 \nu)} k_{1} e^{ \pm \sqrt{-\frac{2 a_{0}^{2}(\sigma-3 \lambda-2 \nu)}{3 \gamma_{k}^{2}}}(k(x-v t))}+k_{2}}\right\} e^{i(-\kappa x+\omega t+\theta)} .
$$


where $a_{0}, v$ and $\omega$ are given by (20), (22) and (23) respectively.

If we set $k_{1}=-\frac{2 a_{0}^{2}(\sigma-3 \lambda-2 \nu)}{3 \gamma k^{2}} e^{ \pm \sqrt{-\frac{2 a_{0}^{2}(\sigma-3 \lambda-2 \nu)}{3 \gamma k^{2}}}} \xi_{0}, \quad k_{2}= \pm 1$, we obtain:

(i) When

$$
\gamma(\sigma-3 \lambda-2 \nu)<0
$$

we have

$$
q(x, t)= \pm a_{0} \tanh \left[\sqrt{-\frac{a_{0}^{2}(\sigma-3 \lambda-2 \nu)}{6 \gamma k^{2}}}\left(k(x-v t)+\xi_{0}\right)\right] e^{i(-\kappa x+\omega t+\theta)}
$$

or

$$
q(x, t)= \pm a_{0} \operatorname{coth}\left[\sqrt{-\frac{a_{0}^{2}(\sigma-3 \lambda-2 \nu)}{6 \gamma k^{2}}}\left(k(x-v t)+\xi_{0}\right)\right] e^{i(-\kappa x+\omega t+\theta)},
$$

These solutions represent dark soliton and singular soliton solutions respectively.

(ii) When

$$
\gamma(\sigma-3 \lambda-2 \nu)>0
$$

we have

$$
q(x, t)= \pm i a_{0} \tan \left[\sqrt{\frac{a_{0}^{2}(\sigma-3 \lambda-2 \nu)}{6 \gamma k^{2}}}\left(k(x-v t)+\xi_{0}\right)\right] e^{i(-\kappa x+\omega t+\theta)},
$$

or

$$
q(x, t)=\mp i a_{0} \cot \left[\sqrt{\frac{a_{0}^{2}(\sigma-3 \lambda-2 \nu)}{6 \gamma k^{2}}}\left(k(x-v t)+\xi_{0}\right)\right] e^{i(-\kappa x+\omega t+\theta)},
$$

These solutions are complex-valued.

\subsection{POWER LAW NONLINEARITY}

The dimensionless form of the perturbed SHE with STD and Power law nonlinearity is given by

$$
i q_{t}+a q_{x x}+b q_{x t}+c|q|^{2 n} q+i\left(\gamma q_{x x x}+\sigma|q|^{2 n} q_{x}\right)=i \alpha q_{x}+i \lambda\left(|q|^{2 n} q\right)_{x}+i \nu\left(|q|^{2 n}\right)_{x} q
$$

Substituting the hypothesis (6) into (33), and and decomposing into real and imaginary parts lead to

$$
\begin{gathered}
k^{2} U^{\prime \prime}(a-b v+3 \gamma \kappa)-\left(a \kappa^{2}+\alpha \kappa+\omega(1-b \kappa)+\gamma \kappa^{3}\right) U+(c-\kappa \lambda+\kappa \sigma) U^{2 n+1}=0, \\
\gamma k^{3} U^{\prime \prime}-k\left(2 a \kappa+\alpha+v(1-b \kappa)-b \omega-3 \gamma \kappa^{2}\right) U+\frac{1}{(2 n+1)} k(\sigma-(2 n+1) \lambda-2 n \nu) U^{2 n+1}=0 .
\end{gathered}
$$

Set

$$
U=V^{\frac{1}{n}}
$$

so that (34) and (35) transform to

$$
\begin{aligned}
& k^{2}(a-b v+3 \gamma \kappa)\left\{n V V^{\prime \prime}+(1-n) V^{\prime 2}\right\}-n^{2}\left(a \kappa^{2}+\alpha \kappa+\omega(1-b \kappa)+\gamma \kappa^{3}\right) V^{2}+ \\
& n^{2}(c-\kappa \lambda+\kappa \sigma) V^{4}=0,
\end{aligned}
$$




$$
\begin{aligned}
& n \gamma k^{3}\left\{n V V^{\prime \prime}+(1-n) V^{\prime 2}\right\}-n^{2} k\left(2 a \kappa+\alpha+v(1-b \kappa)-b \omega-3 \gamma \kappa^{2}\right) V^{2}+ \\
& \frac{n^{2} k}{(2 n+1)}(\sigma-(2 n+1) \lambda-2 n \nu) V^{4}=0 .
\end{aligned}
$$

Balancing $V V^{\prime \prime}$ with $V^{4}$ in Eqs. (37) and (38), then we get $N=1$. Consequently we reach

$$
V(\xi)=a_{0}+a_{1}\left(\frac{\psi^{\prime}(\xi)}{\psi(\xi)}\right), \quad a_{1} \neq 0 .
$$

Substituting Eq. (39) in Eqs. (37) and (38) and then setting the coefficients of $\psi^{-j}(\xi), j=0,1,2,3$, to zero, then we obtain a set of algebraic equations involving $a_{0}, a_{1}, k, \kappa, \gamma, \alpha, \nu, v$ and $\omega$ as follows:

$\psi^{-4}$ coeff.:

$$
\begin{gathered}
a_{1}^{2}\left(\psi^{\prime}\right)^{4}\left(k^{2}(n+1)(a-b v+3 \gamma \kappa)+a_{1}^{2} n^{2}(c+\kappa(\sigma-\lambda))\right)=0, \\
a_{1}^{2} \gamma k^{3} n(n+1)\left(\psi^{\prime}\right)^{4}=0,
\end{gathered}
$$

$\psi^{-3}$ coeff.:

$$
\begin{aligned}
& a_{1}\left(\psi^{\prime}\right)^{2}\left(2 a_{0} n \psi^{\prime}\left(k^{2}(a-b v+3 \gamma \kappa)+2 a_{1}^{2} n(c+\kappa(\sigma-\lambda))\right)-\right. \\
& \left.a_{1} k^{2}(n+2) \psi^{\prime \prime}(a-b v+3 \gamma \kappa)\right)=0, \\
& -\frac{1}{2 n+1} a_{1} k n\left(\psi^{\prime}\right)^{2}\left(a _ { 1 } \left(a_{1} n \psi^{\prime}(\lambda+2 \lambda n+2 \nu n-\sigma)+\right.\right. \\
& \left.\left.\gamma k^{2}\left(2 n^{2}+5 n+2\right) \psi^{\prime \prime}\right)-2 a_{0} \gamma k^{2} n(2 n+1) \psi^{\prime}\right)=0,
\end{aligned}
$$

$\psi^{-2}$ coeff.:

$$
\begin{aligned}
& -3 a_{0} a_{1} k^{2} n \psi^{\prime} \psi^{\prime \prime}(a-b v+3 \gamma \kappa)-a_{1}^{2} n^{2}\left(\psi^{\prime}\right)^{2}\left(-6 a_{0}^{2}(c+\kappa(\sigma-\lambda))+a \kappa^{2}+\alpha \kappa\right. \\
& \left.-b \kappa \omega+\gamma \kappa^{3}+\omega\right)+a_{1}^{2} k^{2}(n-1)\left(\psi^{\prime \prime}\right)^{2}(a-b v+3 \gamma \kappa)-k^{2} n a_{1}^{2} a-b v+3 \gamma \kappa \psi^{\prime} \psi^{\prime \prime \prime}=0, \\
& \frac{1}{2 n+1} k n a_{1}\left(-3 a_{0} \gamma k^{2} n(2 n+1) \psi^{\prime} \psi^{\prime \prime}+a_{1}\left(n \left((2 n+1)\left(-2 a \kappa-\alpha+v(b \kappa-1)+b \omega+3 \gamma \kappa^{2}\right)\right.\right.\right. \\
& \left.n\left((2 n+1)\left(-2 a \kappa-\alpha+v(b \kappa-1)+b \omega+3 \gamma \kappa^{2}\right)\right)\right)=0,
\end{aligned}
$$

$\psi^{-1}$ coeff.:

$$
\begin{gathered}
a_{0} a_{1} n\left(-2 n \psi^{\prime}\left(-2 a_{0}^{2}(c-\kappa \lambda+\kappa \sigma)+a \kappa^{2}+\alpha \kappa-b \kappa \omega+\gamma \kappa^{3}+\omega\right)+\right. \\
\left.k^{2} a-b v+3 \gamma \kappa \psi^{\prime \prime \prime}\right)=0, \\
\frac{a_{0} a_{1} k n^{2}}{2 n+1}\left(\left(-2(2 n+1)\left(2 a \kappa+\alpha-b \kappa v-b \omega-3 \gamma \kappa^{2}+v\right)-\right.\right. \\
\left.\left.3 a_{0}(\lambda+2 \lambda n+2 \nu n-\sigma)\right) \psi^{\prime}+k^{2}(2 n+1) \gamma \psi^{\prime \prime \prime}\right)=0,
\end{gathered}
$$

$\psi^{0}$ coeff.:

$$
\begin{aligned}
& a_{0}^{2}\left(-n^{2}\right)\left(-a_{0}^{2}(c+\kappa(\sigma-\lambda))+a \kappa^{2}+\alpha \kappa-b \kappa \omega+\gamma \kappa^{3}+\omega\right)=0, \\
& -\frac{1}{2 n+1} a_{0}^{2} k n^{2}\left((2 n+1)\left(2 a \kappa+\alpha-b \kappa v-b \omega-3 \gamma \kappa^{2}+v\right)+\right. \\
& \left.a_{0}(\lambda+2 \lambda n+2 \nu n-\sigma)\right)=0,
\end{aligned}
$$


Solving this system, we obtain

$$
\begin{gathered}
a_{1}=0, \quad \omega=\frac{\kappa(\kappa(a+\gamma \kappa)+\alpha)-a_{0}^{2}(c+\kappa(\sigma-\lambda))}{b \kappa-1}, \\
v=\frac{1}{(2 n+1)(b \kappa-1)^{2}}(-(2 n+1)(\kappa(a(2-b \kappa)+\gamma \kappa(4 b \kappa-3))+\alpha)+ \\
\left.a_{0}^{2} b(2 n+1)(c+\kappa(\sigma-\lambda))+a_{0}(b \kappa-1)(\lambda+2 \lambda n+2 \nu n-\sigma)\right)
\end{gathered}
$$

These results lead to the trivial solutions. This is evident from the fact that dark and singular solitons of the type in Kerr law are not supported in power law, which has been proved earlier [6, 10].

\section{CONCLUSIONS}

This paper obtained dark and singular optical soliton solutions to SHE by the aid of modified simple equation method. Both Kerr and power laws of nonlinearity are studied. For power law, it was not possible to retrieve such soliton solutions, as expected. This is because dark and singular solitons of this type ae not supoported in power law medium. This integration scheme fails to obtain bright soliton solutions to SHE since it is an inherent drawback of the algorithm.

This problem will be further studied in future. SHE will be analyed with stochastic as well as time-dependent coefficients. Moreover, the problem will be analyzed with fractional temporal evolution. Additional integration methodology will be employed to obtain further interesting results which will be reported elsewhere.

\section{ACKNOWLEDGEMENTS}

The fourth author (SPM) would like to thank the research support provided by the Department of Mathematics and Statistics at Tshwane University of Technology and the support from the South African National Foundation under Grant Number 92052 IRF1202210126. The fifth author (QZ) was funded by the National Science Foundation of Hubei Province in China under the grant number 2015CFC891. The seventh author (AB) would like to thank Tshwane University of Technology during his academic visit during 2016. The research work of eighth author (MB) was supported by Qatar National Research Fund (QNRF) under the grant number NPRP 6-021-1-005. The authors also declare that there is no conflict of interest. 


\section{References}

[1] A. H. Arnous, M. Mirzazadeh, Q. Zhou, M. F. Mahmood, A. Biswas \& M. Belic. "Optical solitons with resonant nonlinear Schrödinger's equation using $G^{\prime} / G$-expansion scheme". Optoelectronics and Advanced Materials: Rapid Communications. Volume 9, Issue 9-10, 1214-1220. (2013).

[2] A. H. Arnous, M. Mirzazadeh, Q. Zhou, S. P. Moshokoa, A. Biswas \& M. Belic. "Soliton solutions to resonant nonlinear Schrödinger's equation with time-dependent coefficients by modified simple equation method"'. Optik. Volume 127, Issue 23, 11450-11459. (2016).

[3] A. H. Arnous, M. Mirzazadeh, Q. Zhou, S. P. Moshokoa, A. Biswas \& M. Belic. "Optical solitons with higher order dispersions in parabolic law medium by trial solution approach". Optik. Volume 127, Issue 23, 11306-11310. (2016).

[4] I. Bernstein, E. Zerrad, Q. Zhou, A. Biswas, N Melikechi. Dispersive optical solitons with SchrdingerHirota equation by traveling wave hypothesis. Optoelectronics and Advanced Materials: Rapid Communications. Volume 9, Issue 5-6, 792-797. (2015).

[5] I. Bernstein, N. Melikechi, E. Zerrad, Q. Zhou, A. Biswas \& M. Belic. "Dispersive optical solitons in birefringent fibers with Schrödinger-Hirota equation". Journal of Optoelectronics and Advanced Materials. Volume 18, Issues 5-6, 440-444. (2016).

[6] I. Bernstein, N. Melikechi, E. Zerrad, A. Biswas \& M. Belic. "Dispersive optical solitons with Schrödinger-Hirota equation using undetermined coefficients". Journal of Computational and Theoretical Nanoscience. Volume 13, Issue 8, 5288-5293. (2016).

[7] A. H. Bhrawy, A. A. Alshaery, E. M. Hilal, W. Manrakhan, M. Savescu \& A. Biswas. "Dispersive optical solitons with Schrödinger-Hirota equation". Journal of Nonlinear Optical Physics and Materials. Volume 23, Number 1, 1450014. (2014).

[8] A. Biswas. "Optical solitons: Quasistationarity versus Lie transform". Optical and Quantum Electronics. Volume 35, Number 10, 979-998. (2003).

[9] A. Biswas. "Stochastic perturbation of optical solitons in Schrödinger-Hirota equation". Optics Communications. Volume 239, Issues 4-6, 457-462. (2004).

[10] A. Biswas, A. J. M. Jawad, W. N. Manrakhan, A. K. Sarma \& K. R. Khan. "Optical solitons and complexitons of the Schrödinger-Hirota equation". Optics $\&$ Laser Technology. Volume 44, Issue 7, 2265-2269. (2012).

[11] R. K. Dowluru \& P. R. Bhima. "Influences of third order dispersion on linear birefringent optical soliton transmission systems". Journal of Optics. Volume 40, Issue 3, 132-142. (2011).

[12] M. Ekici, M. Mirzazadeh, A. Sonmezoglu, M. Z. Ullah, M. Asma, Q. Zhou, S. P. Moshokoa, A. Biswas \& M. Belic. "Dispersive optical solitons with Schrödinger-Hirota equation by extended trial equation method". Submitted.

[13] M. Eslami, M. A. Mirzazadeh \& A. Neirameh. "New exact wave solution for Hirota equation". Pramana. Volume 84, Number 1, 3-8. (2015).

[14] X. Geng \& Y. Lv. "Darboux transformation for an integrable generalization of the nonlinear Schrödinger equation". Nonlinear Dynamics. Volume 69, Number 4, 1621-1630. (2012). 
[15] S. Kumar, K. Singh \& R. K. Gupta. "Coupled Higgs field equation and Hamiltonian amplitude equation: Lie classical approach and $G^{\prime} / G$-expansion method". Pramana. Volume 79, Number 1, 41-60. (2012).

[16] M. Mirzazadeh, A. H. Arnous, M. F. Mahmood, E. Zerrad \& A. Biswas. "Soliton solutions to resonant nonlinear Schrödinger's equation with time-dependent coefficients by trial solution approach". Nonlinear Dynamics. Volume 81, 277-282. (2015).

[17] M. Mirzazadeh, M. Eslami \& A. H. Arnous. "Dark optical solitons of Biswas-Milovic equation with dual-power law nonlinearity". The European Physical Journal Plus. Volume 130, 1-7. (2015).

[18] P. Wang, B. Tian, W-J. Liu, M. Li \& K. Sun. "Soliton solutions for a generalized inhomogeneous variablecoefficient Hirota equation with symbolic computation". Studies in Applied Mathematics. Volume 125, Issue 2, 213-222. (2010).

[19] E. M. E, Zayed \& A. H. Arnous. "Exact solutions of the nonlinear ZK-MEW and the potential YTSF equations using the modified simple equation method". AIP Conference Proceedings. Volume 1479, 2044-2048. (2012).

[20] E. M. E, Zayed \& A. H. Arnous. "The modified simple equation method and its applications to $(2+$ 1)-dimensional systems of nonlinear evolution equations". Scientific Research and Essays. Volume 8, Issue 40, 1973-1982. (2013). 